\title{
Las arañas en agroecosistemas: bioindicadores terrestres de calidad ambiental
}

\author{
Simó, M. ${ }^{(1) *}$, Laborda, A. ${ }^{(1)}$, Jorge, C. ${ }^{(1)}$, Castro, M. ${ }^{(1)}$ \\ (1) Sección Entomología. Facultad de Ciencias, Universidad de la República, Uruguay. \\ Contacto: simo@fcien.edu.uy \\ Recibido: 23/06/2010 - Aprobado: 28/04/2011
}

\begin{abstract}
$\underline{\text { Resumen }}$
Las arañas son predadores generalistas en ecosistemas terrestres, ampliamente utilizadas en estudios de biodiversidad. En el último siglo, el paisaje de Uruguay se transformó en un mosaico de áreas formadas por ambientes naturales y agroecosistemas. ¿Qué nivel de cambio se espera hallar en la comunidad de arañas si las áreas de estudio están próximas? ¿Qué porcentaje de especies nativas serán reemplazadas con la plantación? ¿Cómo afecta esto a la biodiversidad local? Se realizó un estudio en cuatro áreas vecinas en INIA Las Brujas, Canelones: un bosque ribereño, un bosque de "Espinillo", una plantación de Eucalyptus globulus y una pradera artificial de Trifolium pratense y Avena sativa. Las arañas fueron capturadas con trampas de caída, trampas de tronco, segado, batido de follaje y colecta manual nocturna. Un total de 3.023 adultos fueron colectados. La mayor abundancia y riqueza de especies se observó en el bosque ribereño. A pesar de la proximidad de los sitios estudiados, se observó un alto reemplazo de especies, especialmente entre áreas naturales y agroecosistemas. Los protocolos de colecta utilizados podrían ser útiles para evaluar el impacto de agroecosistemas con diferentes tipos de manejos sobre la biodiversidad local y su aplicación en la certificación de calidad ambiental.
\end{abstract}

Palabras clave: Diversidad, artrópodos, producción agropecuaria, impacto antrópico.

\begin{abstract}
$\underline{\text { Abstract }}$
Spiders are common generalist predators in terrestrial ecosystems, broadly used in diversity studies. The landscape of extensive areas of Uruguay was transformed in a mosaic of patches between natural habitats and agroecosystems. What level of change in the spider community could we expect if these areas are situated near from one another? What percentage of the native species are replaced after the plantation? How this affects the local biodiversity? A study was carried out in four neighboring areas in INIA Las Brujas, Canelones: a riparian forest, an "Espinillo" forest, an Eucalyptus globulus plantation and an artificial prairie of Trifolium pratense and Avena sativa. Spiders were captured with pitfall traps, trunk traps, sweep netting, beating and by nocturnal hand collection. A total of 3.023 adults were collected. Higher values of abundance and species richness were observed in the riparian forest. In spite of the proximity between the studied areas, a high level of replacement of species was observed, especially between natural areas and agroecosystems. The collecting protocols used could be useful to evaluate the impact of differents managements of agroecosystems in the local biodiversity and its application in environmental quality certification. Keywords: Diversity, arthropods, farming production, anthropic impact.
\end{abstract}

\section{Introducción}

Las arañas son artrópodos que constituyen el séptimo grupo en riqueza específica conocida a nivel mundial con unas 42.055 (Platnick, 2011) especies descriptas hasta el momento. Viven en casi todos los ecosistemas terrestres, sin reportes aún en la Antártida. Son predadores generalistas y su dieta está compuesta principalmente por otros artrópodos, tales como insectos y arácnidos. En las últimas décadas se ha estudiado su rol como controlador biológico de plagas en agroecosistemas, teniendo en cuenta la gran abundancia y riqueza específica de arañas en la biomasa animal de invertebrados de los cultivos. Por constituir un grupo megadiverso y ser fáciles de hallar, son sujetos de estudio para la estimación de la diversidad biológica así como en la conservación y calidad medioambiental (Coddington et al., 1996; Cristofoli et al., 2010).

\section{Las arañas como grupo bioindicador}

Un bioindicador es una especie o grupos de especies que refleja el estado abiótico y biótico de un ambiente, es sensible a los cambios ambientales en el ecosistema donde habita y es indicativo de la diversidad de un taxón de un lugar determinado (McGeoch, 1998). Otros criterios utilizados para definir un taxón bioindicador comprenden: buen conocimiento de su taxonomía e historia natural, facilidad para observar y manipular, pertenencia a un grupo con amplia distribución geográfica, sensibilidad a cambios del ambiente y patrones de biodiversidad reflejados sobre otros taxones (géneros o especies) (Pearson, 1994; Halffter et al., 2000). En tanto son predadores generalistas, las arañas se ubican en niveles intermedios de las cadenas tróficas y es importante su rol como predadores de herbívoros (Young y Lockley, 1985). Existen especies de arañas asociadas a ambientes con cierto grado de modificación. Algunas arañas se relacionan con ambientes urbanos y son indicadoras de impacto antrópico, habitan dentro de la vivienda humana o en el peridomicilio. Otras especies se benefician de la actividad humana para ampliar su dispersión geográfica y adaptarse a nuevos ambientes con gran facilidad, constituyéndose en especies introducidas o también invasoras que suelen desplazar a las nativas, generando posteriormente una pérdida de biodiversidad en el ambiente. Por tanto, desde hace un tiempo existe gran interés en la utilización de las arañas para el monitoreo ambiental y para el establecimiento de medidas de prevención (Taylor y Doran, 2001). La identificación de especies indicadoras requiere de estudios previos básicos de 
diversidad biológica. En el caso de Uruguay la araneofauna de muchos ambientes nativos del país es desconocida, al igual que la de zonas con mayor impacto humano.

\section{Las arañas en agroecosistemas}

El estudio de las arañas en agroecosistemas se desarrolló a partir del interés que plantea este grupo como posibles controladores de plagas (Nyffeler y Sutherland, 2003). Los grupos funcionales o gremios representan diferentes grupos de arañas que disponen de determinados mecanismos para la obtención de los recursos. En los estudios de la araneofauna de agroecosistemas, la identificación de los gremios predominantes permite conocer las principales estrategias de captura de la comunidad y en particular de aquella que resulta importante para establecer el control biológico. Las arañas tejedoras representan una gran parte de la comunidad de un cultivo y son las mejores a la hora de seleccionar un controlador biológico. Pero existen otros gremios como las arañas errantes del suelo, las errantes de vegetación y las cazadoras al acecho en vegetación que conforman la comunidad de arañas.

La superficie de Uruguay, sobre la que se realiza una gran producción agropecuaria, representa un mosaico de agroecosistemas. El relativamente pequeño tamaño y la alta diversidad paisajística convierten al país en un lugar muy acorde para estudiar la araneofauna en diversos ambientes. El primer estudio de arañas en agroecosistemas en Uruguay fue realizado en la década de 1990 en una huerta orgánica de Montevideo (Viera y Simó, 1996) y posteriormente se realizó otro en plantaciones de limón (Benamú Pino, 2005); ambos apuntaron a indicar las especies presentes en dichos ambientes. El desarrollo de la aracnología en el país en los últimos 10 años, así como el avance de la investigación científica basado en planes de posgrado ha llevado a que el estudio de las arañas en agroecosistemas se constituya en breve en una de las principales líneas de investigación en este grupo. Teniendo en cuenta que las arañas componen un grupo megadiverso, con metodologías relativamente sencillas para el muestreo y con una importante abundancia en las colectas, es posible emplearlas como grupo o taxa indicador de la biodiversidad local o regional. Esto permite evaluar de qué forma una práctica agrícola puede incidir en mayor o menor grado con el mantenimiento de la diversidad local de arañas y ser un elemento medible y comparativo para estimar la conservación de la biodiversidad (Haughton et al., 1999; Pinkus et al., 2006).

\section{Análisis de un estudio realizado en INIA Las} Brujas

Tomando como punto de partida el mosaico de ambientes naturales y agroecosistemas que presenta Uruguay, se realizó un estudio para conocer el grado de recambio en la araneofauna producida por los agroecosistemas sobre los ambientes naturales circundantes. Las preguntas respondidas fueron: ¿qué grado de reemplazo de especies de arañas presentaban entre sí estos cuatro ambientes? y ¿qué especies se pueden considerar más representativas de la taxocenosis en los agroecosistemas?

\section{Materiales y Métodos}

El estudio se realizó en un área de amortiguación del Parque

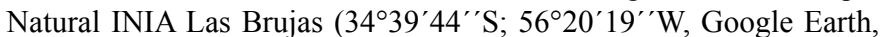
2009), entre 2004 y 2005, enmarcado en la zona de conservación de los Humedales del Santa Lucía.

Se analizaron dos áreas naturales, testigos del ambiente original de la zona, un bosque ribereño y un área de bosque parque de Acacia caven (Espinillo) y dos agroecosistemas contiguos: una pradera artificial (Avena sativum y Trifolium pratense) y una plantación de Eucalyptus globulus de 11 años (Figuras 1 y 2).

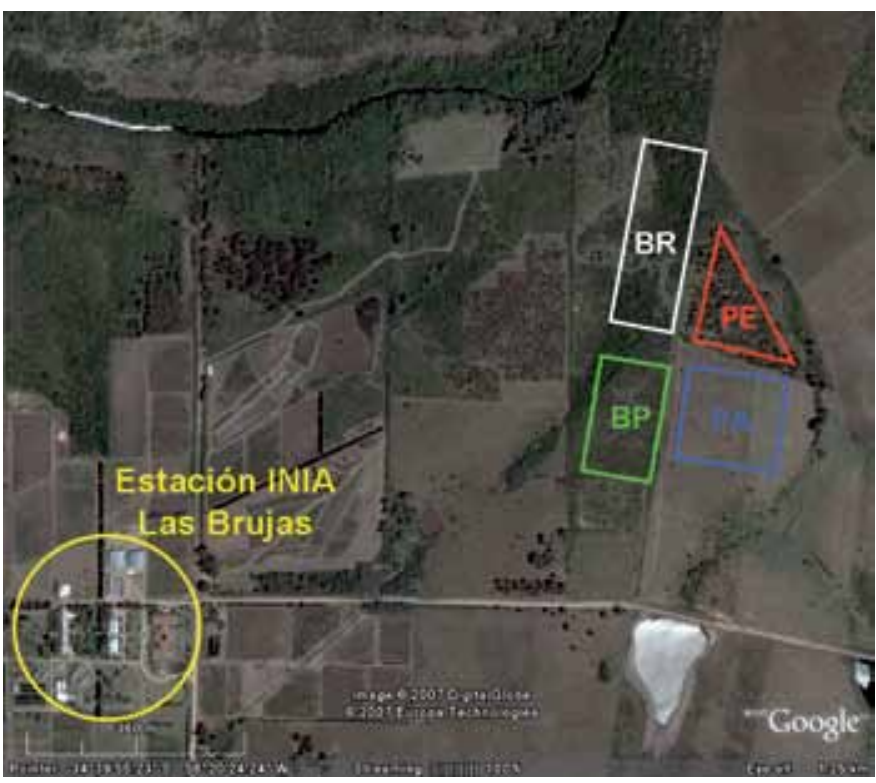

Figura 1. Imagen aérea de las zonas de muestreo. PA: plantación de avena; PE: plantación de Eucalyptus globulus; BP: bosque parque "Espinillar" y BR: bosque ribereño.

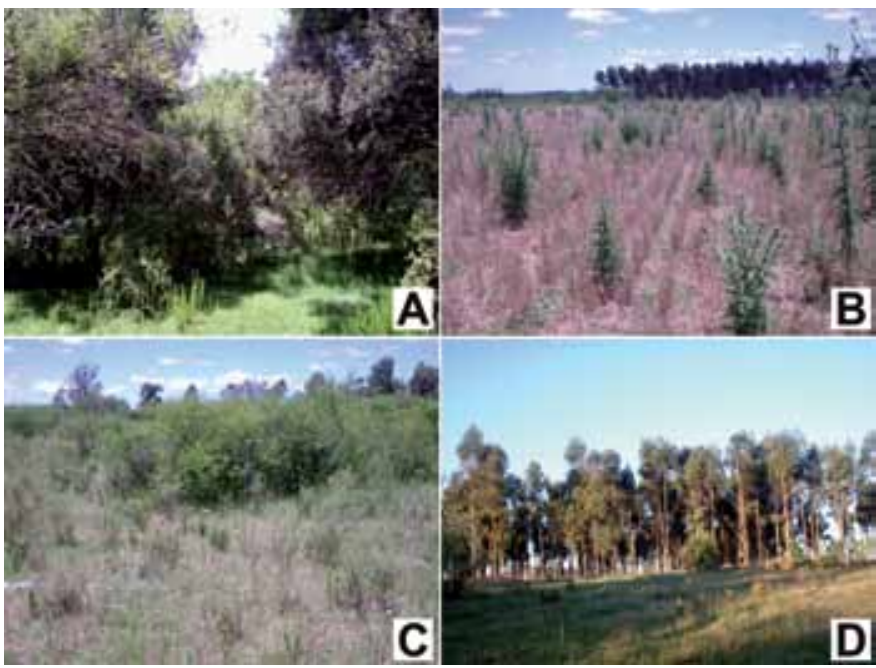

Figura 2. Vistas de las zonas de estudio. A: bosque ribereño; B plantación de avena; C: bosque parque; D: plantación de Eucalyptus globulus.

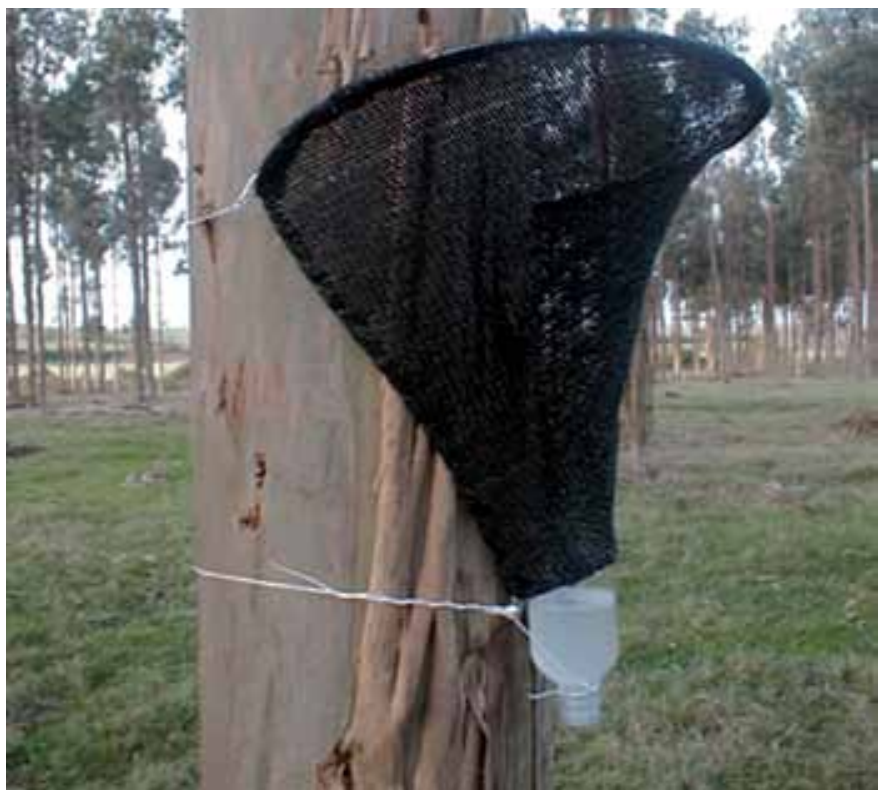

Figura 3. Trampa de tronco en Eucalyptus globulus. 
El estudio abarcó muestreos en las cuatro estaciones del año. En cada muestreo se emplearon métodos de recolección directos e indirectos. Los directos consistieron en 10 muestras de 10 pases de segado con red entomológica, 10 recolecciones de batido de follaje con una intensidad de 10 golpes por muestra y colecta manual nocturna durante 15 minutos en la que participaron 3 a 4 recolectores. Los métodos indirectos empleados fueron: 5 trampas de caída ubicadas cada 10 metros en cada zona de estudio y 5 trampas de tronco en la plantación de Eucalyptus y en el bosque ribereño. Las trampas fueron controladas cada 15 días durante todo el período de estudio. Se calcularon la riqueza, equitatividad y diversidad de cada área. La riqueza se evaluó en base al número de especies constatadas. La equitatividad se estableció de acuerdo a la proporción relativa en la que cada una de las especies está presente en cada sitio estudiado. La diversidad fue analizada mediante el índice de Shannon, el cual expresa un valor que relaciona el número de especies con sus abundancias relativas. Para el cálculo de estos valores se utilizó el programa Biodiversity Pro 2.0 (Mc Aleece, 1997). La similaridad de la araneofauna entre las zonas de estudio fue analizada mediante el índice de Jaccard con el programa Simil \& Divers (Franja, 1993). Este índice estima la similaridad cualitativa en base a la presencia de especies compartidas entre las zonas de estudio.

\section{Resultados}

Las mayores abundancias de arañas se registraron en el bosque ribereño y la pradera artificial (Figura 4). En cuanto a la riqueza específica, el bosque ribereño presentó el valor más alto (86 especies) y el menor correspondió al bosque parque (60 especies). Los mayores valores de equitatividad se registraron en la plantación de $E$. globulus (0.77) y en el bosque parque (0.72), y las menores en el bosque ribereño (0.63) y en la pradera artificial (0.61).

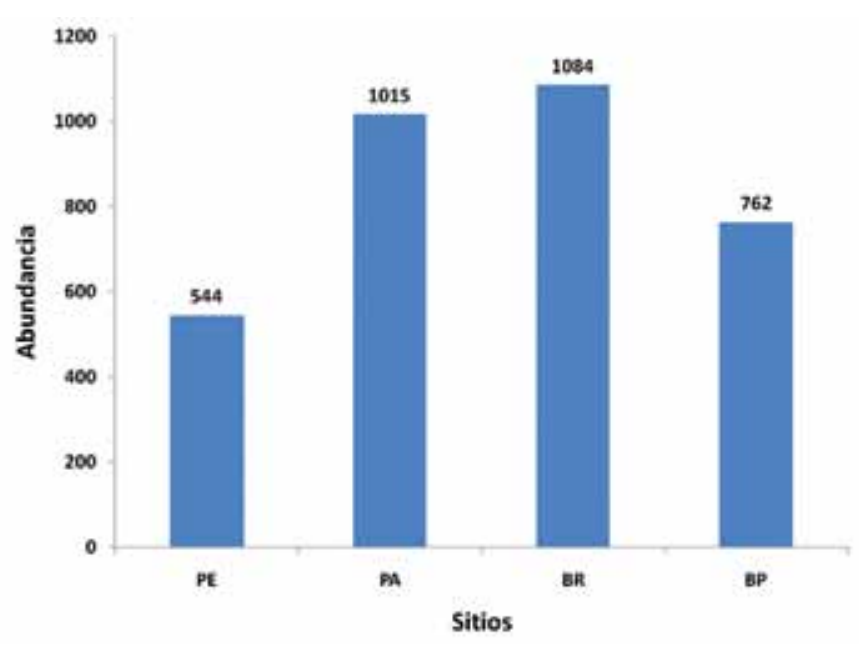

Figura 4. Abundancias de las arañas por zonas de muestreo. BR bosque ribereño; BP: bosque parque "Espinillar"; PE: plantación de Eucalyptus globulus; PA: plantación de pradera artificial (Avena sativa y Trifolium pratense).

El bosque ribereño mostró también una mayor predominancia del gremio de las arañas tejedoras; las de telas irregulares fueron las que presentaron mayor número de especies en todos los ambientes estudiados.

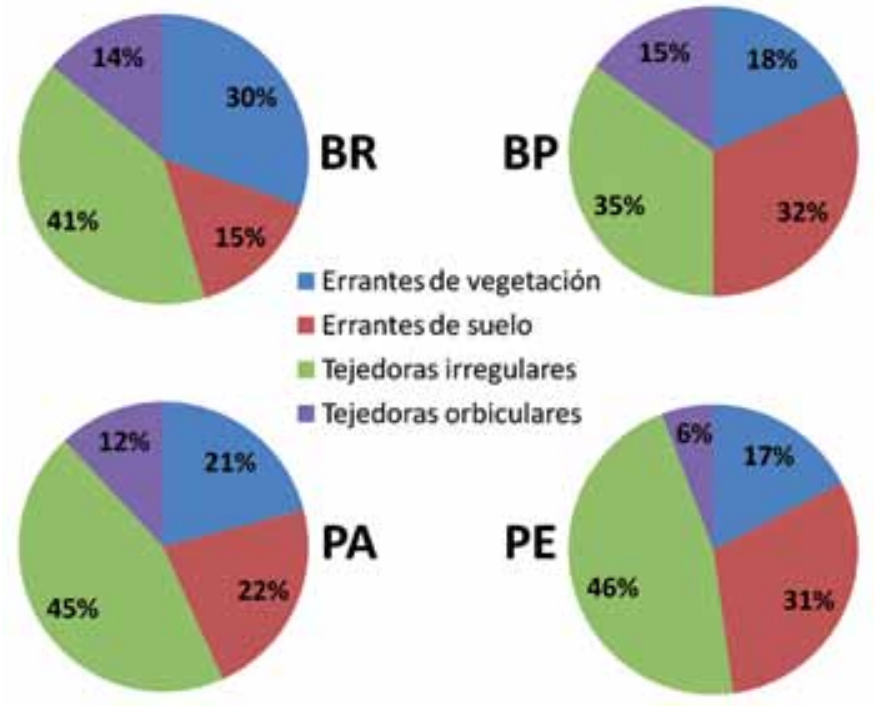

Figura 5. Representación de los gremios de arañas en cada una de las zonas de estudio. BR: bosque ribereño. BP: bosque parque "Espinillar". PE: plantación de Eucalyptus globulus. PA: plantación de pradera artificial (Avena sativa y Trifolium pratense).

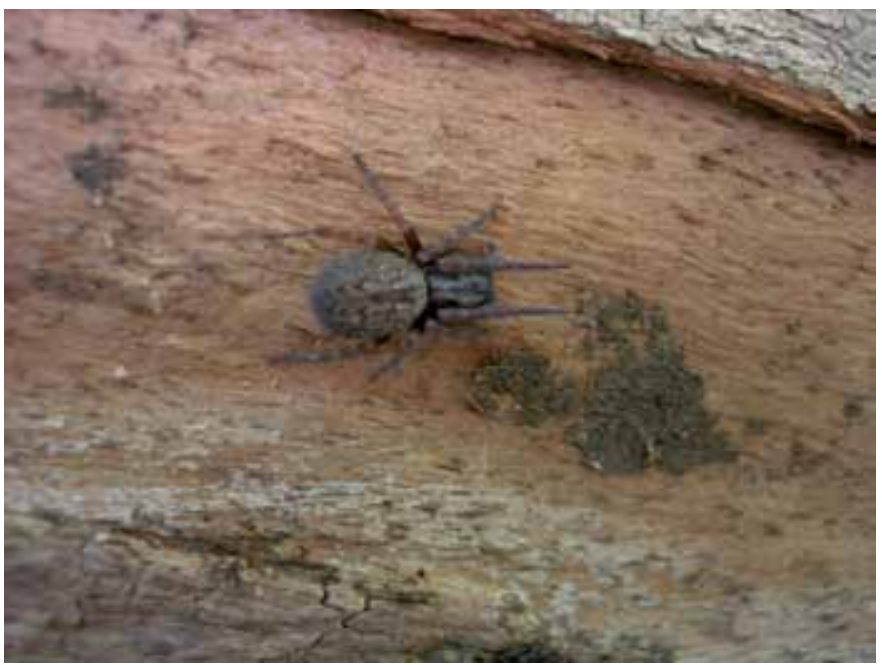

Figura 6. Badumna longinqua sobre tronco de Eucalyptus globulus.

De un total de 3.023 arañas adultas recolectadas, Badumna longinqua (L. Koch, 1867) (Desidae) se halló exclusivamente sobre los troncos de la plantación de Eucalyptus. Otra especie común en los troncos fue Polybetes pythagoricus (Holmberg, 1875) (Sparassidae) y Scolecura propincua (Millidge, 1991) (Linyphiidae) fue abundante sobre el suelo en la plantación. En la pradera artificial, Oxyopes salticus (Hentz, 1845) fue una de las especies mejor representadas en el muestreo.

La Tabla 1 expresa que la similaridad entre los ambientes estudiados, basada en datos cualitativos de ausencia y presencia, fue relativamente baja, ubicada entre el $24 \%$ y $43 \%$. 


\begin{tabular}{|c|c|c|c|}
\hline Jaccard & BP & PA & PE \\
\hline BR & $\mathbf{0 , 3 3}$ & $\mathbf{0 , 2 4}$ & $\mathbf{0 , 3 6}$ \\
\hline BP & & $\mathbf{0 , 3 7}$ & $\mathbf{0 , 4 0}$ \\
\hline PA & & & $\mathbf{0 , 4 3}$ \\
\hline
\end{tabular}

Tabla 1. Índice de similaridad de Jaccard entre las zonas de estudio. Abreviaturas: BP: Bosque parque; PA: plantación de Avena sativum; PE: plantación de Eucalyptus globulus; BR: bosque ribereño.

\section{Discusión y Conclusiones}

Estudios como el de Avalos et al. (2007) han demostrado que las arañas son buenos indicadores de impacto ambiental, debido a su sensibilidad a los cambios ambientales naturales y antrópicos. La sustitución de los ambientes naturales por agroecosistemas genera un recambio de especies en la comunidad de arañas local. En el estudio desarrollado, el bosque ribereño presentó el mayor número de especies, por lo que su conservación permitiría contribuir a mantener la diversidad local de este grupo. El establecimiento de medidas de conservación de los ambientes naturales de la zona de estudio, en este caso el bosque ribereño y el bosque parque de Espinillar, sería una estrategia adecuada para la conservación de la diversidad biológica local, tomando como grupo indicador las arañas. La mayor riqueza específica hallada en el bosque ribereño, así como el predominio de arañas tejedoras serían debidas a la mayor heterogeneidad espacial que presenta este ambiente nativo. Esta cualidad propicia la presencia de una mayor oferta de microhábitats, mayor soporte y refugio para el establecimiento de las especies. La mayor diversidad hallada en la plantación de E. globulus se explicaría por tratarse de la zona con mayor valor de equitatividad.

La araña pollito Acanthoscurria suina Pocock, 1903 que vive en nidos subterráneos (Pérez-Miles et al., 2005), sólo se encontró en los ambientes naturales. Su ausencia en los cultivos podría deberse a los cambios del suelo, como la presencia del manto de hojarasca en la plantación de E. globulus, o a su sensibilidad a los manejos agrícolas, como la acción de rotativas en la pradera artificial. Debido a ello esta especie podría ser considerada como buen indicador de impacto antrópico. Si bien las áreas cultivadas comparten un porcentaje de especies en común con las áreas naturales contiguas, se apreció que los cultivos permiten el establecimiento de nuevas especies como respuesta al cambio ambiental. Este estudio posibilitó conocer que el mosaico de ambientes estudiados, a pesar de ubicarse contiguos unos con otros, presentó una baja similaridad y, por tanto, un alto reemplazo de especies entre ellos. A pesar de diferir en el porte vegetal, la mayor similaridad a nivel de la araneofauna hallada entre las plantaciones de E. globulus y la pradera artificial sería respuesta a la presencia de especies asociadas al impacto humano. Oxyopes salticus fue una de las especies mejor representadas en el estrato herbáceo de las zonas estudiadas. Esta especie ha sido reportada como muy importante en el control de plagas agrícolas, especialmente estadios larvales de Lepidópteros en cultivos de algodón, soja, sorgo, alfalfa y arroz, y ha demostrado ser resistente a la aplicación de algunos insecticidas (Young y Lockley, 1985).

La presencia de dos arañas invasoras como B. longinqua y Ostearius melanopygius (Linyphiidae) en los agroecosistemas representa la colonización por especies oportunistas que pueden llegar a competir con las especies nativas, por lo que sería de interés su monitoreo y control. La modificación de ciertas prácticas en los cultivos permitiría minimizar el efecto negativo sobre la diversidad local. Estudios futuros podrían estar enfocados al posible papel de los agroecosistemas como reservorios de especies de arañas nativas y corredores faunísticos entre los ambientes naturales. De esta manera el conocimiento de la comunidad de arañas puede ser utilizado para evaluar el impacto que los agroecosistemas producen en las comunidades biológicas nativas y constituirse en nuevos recursos de aporte de información para la certificación ambiental de las prácticas agrícolas.

\section{Reconocimientos}

Al Ing. Agrónomo Oscar Blumetto de la Estación INIA Las Brujas por el apoyo al proyecto y en las gestiones para los estudios a campo. A la flia. Fernández, propietaria de uno de los sitios donde se realizaron los muestreos. A la Prof. Mag. Lucía Rodríguez por su ayuda para la elección del área de estudio. A Mónica Rodríguez, María Eugenia Rodríguez, Nadia Falero, Marcos Llhano, José Rodríguez y Clemente Olivera por la colaboración en los trabajos de campo. A Marcelo Dias y a un revisor anónimo por los aportes y comentarios al manuscrito.

\section{Referencias}

\section{- AVALOS, G.; RUBIO, G.; BAR, M; GONZÁLEZ, A. Arañas} (Arachnida: Araneae) asociadas a dos bosques degradados del Chaco húmedo en Corrientes, Argentina. En: Revista de Biología Tropical. 2007, 55(3-4):899-909.

- BENAMÚ PINO, M. A. Estudio comparativo de la diversidad de arañas de un campo en abandono y un cultivo convencional de limonero (Citrus limon [L.] Burm.) en Rincón del Cerro, Montevideo, Uruguay. Montevideo: Facultad de Ciencias; PEDECIBA, 2005. (Tesis de Maestría).

- CODDINGTON, J.; YOUNG, L.; COYLE, F. Estimating spider species richness in a Southern Appalachian Cove Hardwood Forest. En: Journal of Arachnology. 1996, 24(2):111-125.

- CRISTOFOLI, S.; MAHY, G.; KEKENBOSCH, R.; LAMBEETS, K. Spider communities as evaluation tools for wet heathland restoration. En: Ecological Indicators. 2010, 10:773-780.

- FRANJA. Programa índice de diversidad (divers.exe) y medidas de similitud (simil.exe) [En línea]. [s.1.]: Franja, 1993. [Consulta 4 de marzo de 2010]. Disponible en: http//www.perso.wanadoo.es/ Jp-L/descargas.html

- GOOGLE INC. Google Earth (Version 5.0.11733.9347) [En línea]. [s.1.]: Google Inc, 2009. [Consulta: 3 de diciembre de 2009]. Disponible en: http://earth.google.es/download-earth.html

- HALFFTER, G.; MORENO, C. L.; PINEDA, E. O. Manual para evaluación de la biodiversidad en Reservas de la Biosfera. En: Manuales \& Tesis. 2001, 2:1-77.

- HAUGHTON, A.; BELL, J.; BOATMAN, N.; WILCOX, A. The effects of different rates of the herbicide glyphosate on spiders in arable field margins. En: The Journal of Arachnology. 1999, 27:249-254.

- MC ALEECE, N. Biodiversity Professional 2.0. [En línea]. [s.1.]: The Natural History Museum; The Scottish Association for Marine Science, 1997. [Consulta 4 de marzo de 2010]. Disponible en: http://www.sams.ac.uk/research/software

- MCGEOCH, M. A. The selection, testing and application of terrestrial insects as bioindicators. En: Biological Reviews. 1998, 73:181- 201.

\section{- NYFFELER, M.; SUTHERLAND, K. Composition,}

abundance and pest control potential of spider communities in agroecosystems: a comparison of European and U. S. studies. En: Agriculture Ecosystems Environment. 2003, 95:579-612. 
- PEARSON, D.L. Selecting Indicator Taxa for the quantitative assessment of Biodiversity. En: Philosophical Transaction of The Royal Society of London, Series B : Biological Sciences. 1994, 345 :75 -79.

- PÉREZ-MILES, F.; COSTA, F. G.; TOSCANO-GADEA, C.; MIGNONE, A. Ecology and behaviour of the "road tarantulas" Eupalaestrus weijenberghi and Acanthoscurria suina (Araneae, Theraphosidae) from Uruguay. En: Journal of Natural History. 2005, 39(6):483-498.

- PINKUS RENDÓN, M.; IBARRA-NÚÑEZ, G.; PARRA-TABLA, V.; GARCÍA-BALLINAS, J.; HÉNAUT, Y. Spider diversity in coffee plantations with different management in Southeast Mexico. En: The Journal of Arachnology. 2006, 34:104-112.

- PLATNICK, N. I. The World spider catalog, version 11.5 [En línea]. New York: American Museum of Natural History, 2011. [Consulta: febrero, 2011]. Disponible en: http://research.amnh.org/ entomology/spiders/catalog/index.html

- TAYLOR, R. J.; DORAN, N. Use of terrestrial invertebrates as indicators of the ecological sustainability of forest management under the Montreal Process. En: Journal of Insect Conservation. 2001, 5:221-231.

- VIERA, C.; SIMÓ, M. La comunidad de arañas epigeas de un agroecosistema orgánico de Uruguay (Araneae). Comunicación Preliminar. En: Actas Resúmenes $21^{\circ}$ Congresso Brasileiro de Zoología. Porto Alegre: Brasil, 1996. pp. 35.

- YOUNG, O. P.; LOCKLEY, T. The striped lynx spider, Oxyopes salticus (Araneae : Oxyopidae), in agroecosystems. En: Entomophaga. 1985, 30(4):329-346. 